\title{
Comparing the spectral lag of short and long gamma-ray bursts and its relation with the luminosity
}

\author{
Maria Grazia Bernardini* ${ }^{* \dagger}$ \\ INAF - Osservatorio Astronomico di Brera \\ E-mail: grazia.bernardini@brera.inaf.it
}

\section{Giancarlo Ghirlanda}

INAF - Osservatorio Astronomico di Brera

We present the analysis of the rest frame spectral lags of two complete samples of bright long (50) and short (6) gamma-ray bursts (GRB) detected by Swift. We find that half of the long GRBs have a positive lag and half a lag consistent with zero. All short GRBs have lags consistent with zero. The distributions of the spectral lags for short and long GRBs have different average values. Limited by the small number of short GRBs, we cannot exclude at more than 2 sigma significance level that the two distributions of lags are drawn from the same parent population. If we consider the entire sample of long GRBs, we do not find evidence for a lag-luminosity correlation, rather the lag-luminosity plane appears filled on the left hand side, thus suggesting that the lag-luminosity correlation could be a boundary. Short GRBs are consistent with the long ones in the lag-luminosity plane.

Swift: 10 Years of Discovery

2-5 December 2014

La Sapienza University, Rome, Italy

\footnotetext{
${ }^{*}$ Speaker.

${ }^{\dagger}$ The author acknowledge support from ASI-INAF I/088/06/0 and PRIN-INAF 1.05.01.09.15 grants.
} 


\section{Introduction}

The presence of a positive spectral lag (i. e. a delay in the arrival times of low-energy photons with respect to high-energy photons) was identified by extensive studies of long gamma-ray burst (GRB) prompt emission observed by BATSE [1]. Besides, it has been found that this positive spectral lag for long GRBs is anti-correlated with the burst bolometric peak luminosity [5]. Since then, it has been considered a distinctive feature of long GRB prompt emission, and it has been used as a possible tool to discriminate between long and short GRBs [2], since the latter tend to have a smaller lag (consistent with zero) with respect to long GRBs [3, 4]. The anti-correlation has been also explored and confirmed with different samples, however with limited sizes [6, 7, 8, 9, 10].

The large increase in the redshift measurements available thanks to the advent of Swift [11] allowed Ukwatta et al. (2012 [12]) to perform the first detailed analysis of the spectral lag for long GRBs, adopting two selected rest frame energy bands for all the considered burst. They confirmed the existence of the correlation with a smaller scatter when compared to previous analyses in the observer-frame [10]. However, in the determination of the lag-luminosity correlation they did not consider $44 \%$ of the GRBs of their original sample that have spectral lag consistent with zero or negative. The restriction to the GRBs with a spectral lag greater than zero and the consequent exclusion of about a half of the total sample introduces a bias: since the physical origin of the spectral lag and of this correlation is not well understood, there is no a priori reason to consider lags consistent with zero or negative as spurious.

Here we review the main results of a comprehensive analysis of the spectral lag for both long and short GRBs presented in Bernardini et al. (2015 [13]), whose aim is to investigate the opportunity to use it as a distinctive feature for these two classes of GRBs, and the role of negligible lags of long GRBs in the lag-luminosity plane.

\section{Sample selection and methodology}

We investigated the rest frame spectral lag $\left(\tau_{R F}=\tau /(1+z)\right)$ for both long and short GRBs. We make use of two complete samples of bright long (50) and short (6) GRBs detected by Swift/Burst Alert Telescope (BAT, [14]) presented in Salvaterra et al. (2012 [15]; BAT6) and in D'Avanzo et al. (2014 [16]; S-BAT4) to constrain the properties of the spectral lag. Since the spectral lag is dependent upon the energy bands chosen to compute it, we adopted two fixed rest-frame energy bands $(100-150 \mathrm{keV}$ and $200-250 \mathrm{keV})$ to perform a direct comparison of the lags of the two classes of long and short GRBs.

With the background subtracted light curves observed by the Swift/BAT we computed the discrete cross-correlation function (CCF) to measure the temporal correlation of the two light curves in the two different energy bands. The CCF is modelled with an asymmetric Gaussian model to search for its global maximum that, by definition, corresponds to the spectral lag $\tau$. We choose an asymmetric Gaussian model since it reflects the natural asymmetry of the CCF inherited by the asymmetry of the GRB pulses. We accounted for the errors on the data points through a Monte Carlo method to estimate the uncertainty on $\tau$ [13]. 


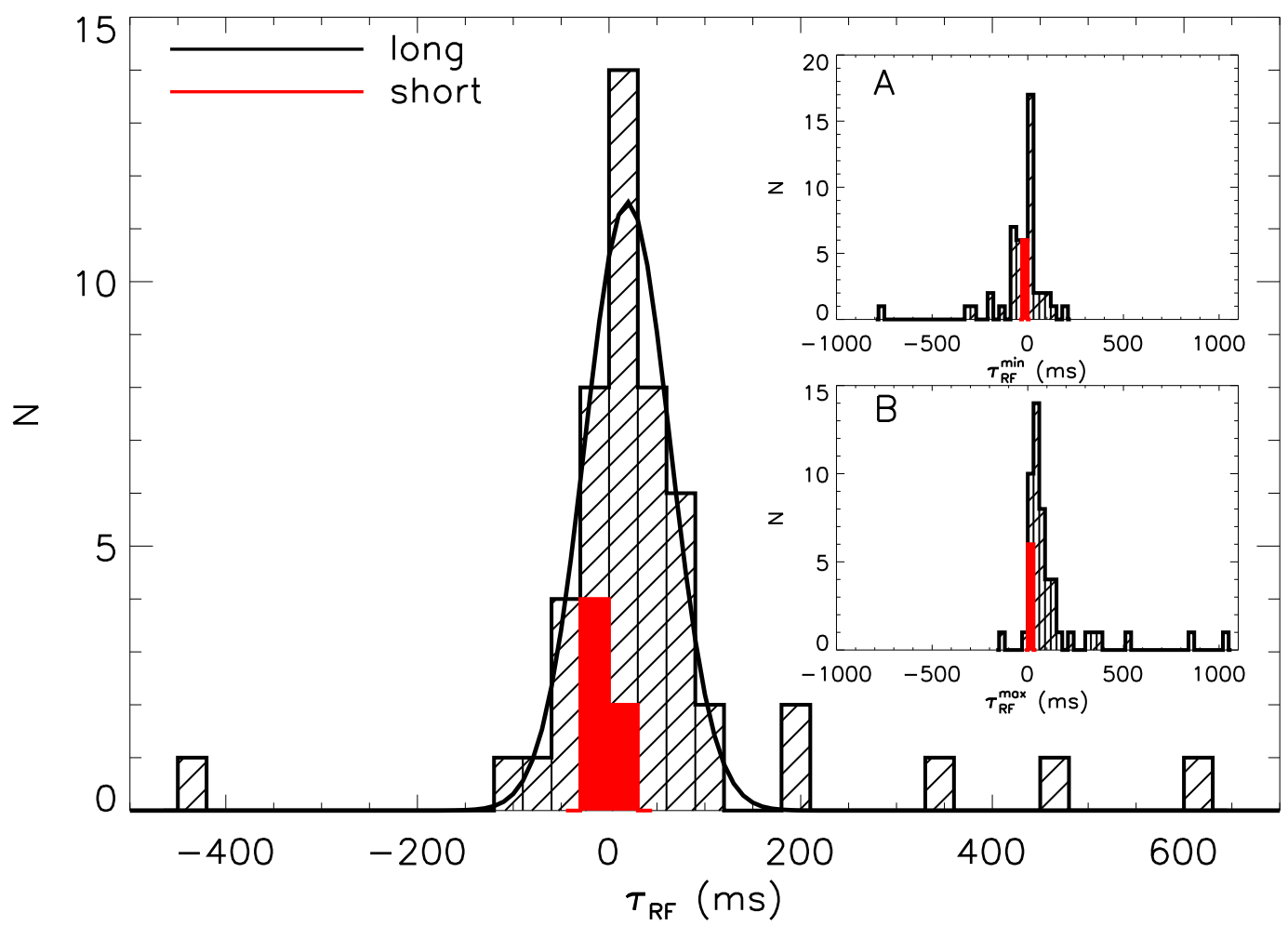

Figure 1: Mean values of the rest frame spectral lags. The black solid line is a gaussian fit to the distribution for the long GRBs $(\mu=19.2, \sigma=44.4, N=11.5)$. Inset A: minimum rest frame spectral lag, defined as $\tau_{\mathrm{RF}}^{\min }=\tau_{\mathrm{RF}}-\sigma_{\mathrm{l}, \mathrm{RF}}$. Inset B: maximum rest frame spectral lag, defined as $\tau_{\mathrm{RF}}^{\max }=\tau_{\mathrm{RF}}+\sigma_{\mathrm{r}, \mathrm{RF}}$.

\section{The spectral lag of short and long GRBs}

We found that the spectral lag between the chosen rest frame energy bands for long GRBs is significantly (within $1 \sigma$ ) greater than zero in most cases (50\%). However an equally large fraction $(50 \%)$ of them are consistent with zero or negative within errors. Short GRBs have in all cases limited or no lag in the same rest frame energy bands [13].

The distribution of the spectral lags for short GRBs is peaked at a smaller value than the long GRB distribution (mean value of the distribution $\left\langle\tau_{R F}^{S}\right\rangle=(-0.61 \pm 3.87)$ ms compared to $\left\langle\tau_{R F}^{L}\right\rangle=(43.0 \pm 17.8) \mathrm{ms}$, see fig. 1). However, there is no stronger than $2 \sigma$ statistical indication that the spectral lags of short and long GRBs are drawn from two different populations: if we perform a Kolmogorov-Smirnov (KS) test, the probability that the two samples are drawn from the same population is $4.1 \%$. The time-integrated spectral lag as a tool to distinguish between short and long GRBs might not be as definite as thought before: the existence of a large fraction of long GRBs with a lag consistent with zero makes it challenging to classify the ambiguous GRBs.

\section{The lag-luminosity correlation for long and short GRBs}

We considered all the GRBs with measured lags in our samples (long and short) that also have an estimate of the bolometric isotropic luminosity $L_{\text {iso }}$ to investigate the relation between the 


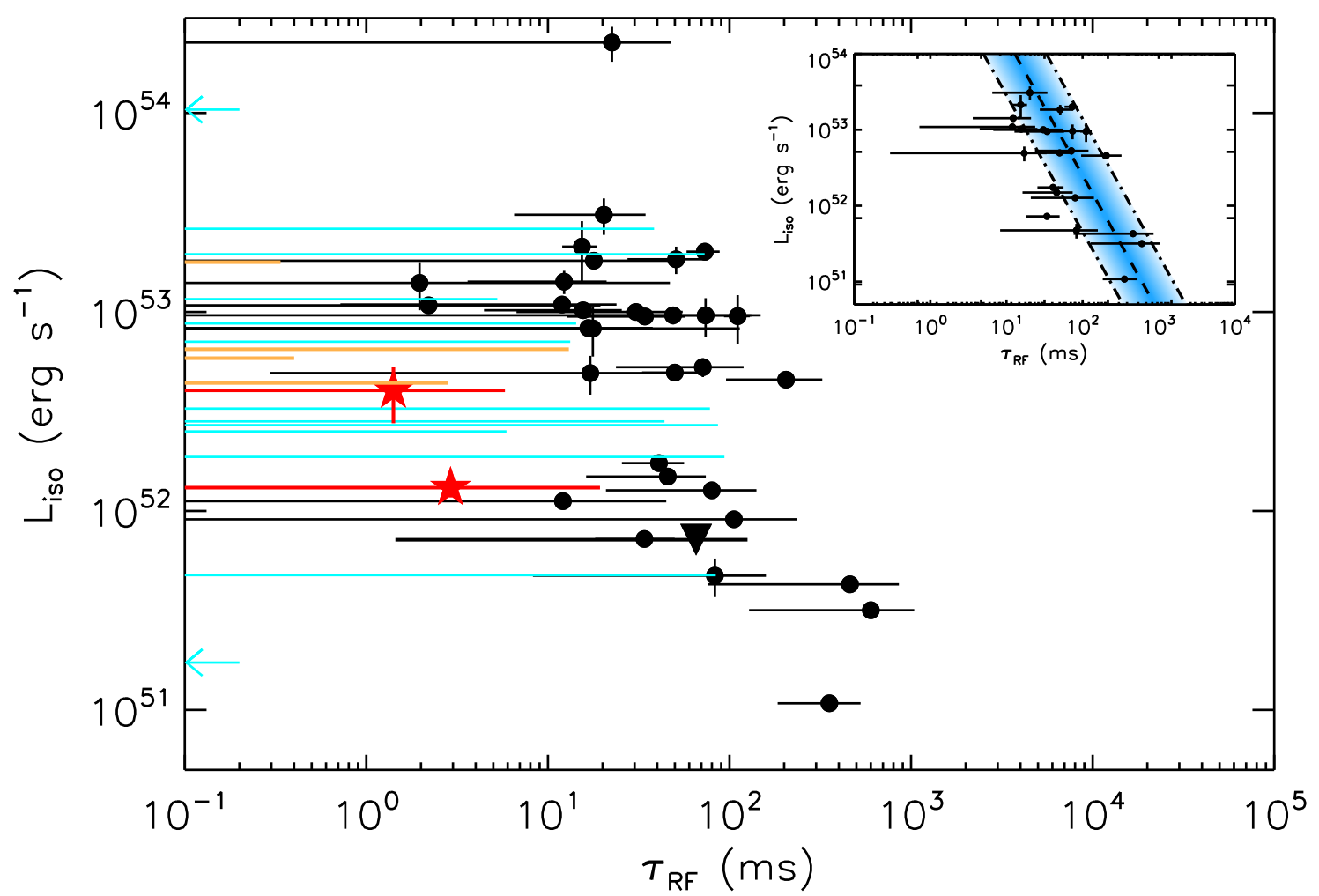

Figure 2: Peak luminosity $L_{\text {iso }}$ as a function of the rest-frame spectral lag. Black points: long GRBs with positive central value of the spectral lag (23 with positive lag and 9 with positive lag consistent with zero within errors). Cyan points: long GRBs with negative central value of the spectral lag (2 with negative lag, marked as left arrows, and 11 with negative lag consistent with zero within errors). Red stars: short GRBs with positive central value of the spectral lag ( 2 with positive lag consistent with zero within errors). Orange stars: short GRBs with negative central value of the spectral lag (4 with negative lag consistent with zero within errors). The black triangle corresponds to GRB 100816A. Inset: lag-luminosity anti-correlation for the 23 long GRBs with positive lag. The black dashed line is the best fit to the data: $\log \left[L_{\text {iso }} /\left(10^{52} \mathrm{erg} \mathrm{s}^{-1}\right)\right]=$ $(0.42 \pm 0.11)+(-1.79 \pm 0.03) \log \left[\tau_{R F} / 100 \mathrm{~ms}\right]$, and the blue area marks the $1-\sigma$ region around the best fit.

spectral lag and the GRB luminosity, namely 45 long GRBs and 6 short GRBs ${ }^{1}$.

If we restrict our analysis to all long GRBs with positive spectral lag (23; $51 \%$ of the sample), we find that the luminosity significantly anti-correlates with the spectral lag (Pearson correlation coefficient $r=-0.68$, null-hypothesis probability $P=3.8 \times 10^{-4}$ ). The best linear fit to the $\log \left[L_{\text {iso }}\right]-\log \left[\tau_{R F}\right]$ correlation that accounts for the statistical uncertainties on both axes yields: $\log \left[L_{\text {iso }} /\left(10^{52} \mathrm{erg} \mathrm{s}^{-1}\right)\right]=(0.42 \pm 0.11)+(-1.79 \pm 0.03) \log \left[\tau_{R F} / 100 \mathrm{~ms}\right]$ (see fig. 2 , inset). The scatter perpendicular to the correlation is modelled with a Gaussian with standard deviation $\sigma=0.65$.

However, when we add to the lag-luminosity plane also the 20 long GRBs with lag consistent with zero within errors, no correlation between $L_{\text {iso }}$ and the spectral lag is anymore apparent (see fig. 2). There are also two long GRBs with negative lag (GRB 061021 and GRB 080721; cyan

\footnotetext{
${ }^{1}$ For the values of $L_{\text {iso }}$ and its definition we refer to [17] and [16].
} 
arrows in fig. 2). The lag-luminosity correlation appears as a boundary in the plane, challenging the possible use of this relation for cosmological purposes.

Short GRBs of our sample do not occupy a separate region of the lag-luminosity plane when compared to the total sample of long GRBs (see fig. 2), because neither their distribution of the spectral lag is significantly different from the long GRB one, nor the luminosity distributions (KS probability $P=38 \%$ ). Therefore, the lag-luminosity correlation is questioned by these findings.

\section{References}

[1] Cheng, L. X., Ma, Y. Q., Cheng, K. S., Lu, T., Zhou, Y. Y., The time delay of gamma-ray bursts in the soft energy band, A\&A 300 (1995) 746.

[2] Gehrels, N. et al., A new $\gamma$-ray burst classification scheme from GRB060614, Nature 444 (2006) 1044.

[3] Norris, J. P., Scargle, J. D., Bonnell, J. T., Short Gamma-Ray Bursts Are Different In: Costa, E., Frontera, F., Hjorth, J. (Eds.), Gamma-ray Bursts in the Afterglow Era (2001).

[4] Norris, J. P., Bonnell, J. T., Short Gamma-Ray Bursts with Extended Emission, ApJ 643 (2006) 266.

[5] Norris, J. P., Marani, G. F., Bonnell, J. T., Connection between Energy-dependent Lags and Peak Luminosity in Gamma-Ray Bursts, ApJ 534 (2000) 248.

[6] Norris, J. P., Implications of the Lag-Luminosity Relationship for Unified Gamma-Ray Burst Paradigms, ApJ 579 (2002) 386.

[7] Schaefer, B. E., The Hubble Diagram to Redshift greater than 6 from 69 Gamma-Ray Bursts, ApJ 660 (2007) 16.

[8] Hakkila, J., Giblin, T. W., Norris, J. P., Fragile, P. C., Bonnell, J. T., Correlations between Lag, Luminosity, and Duration in Gamma-Ray Burst Pulses, ApJ Lett. 677 (2008) L81.

[9] Arimoto, M. et al., Spectral-Lag Relations in GRB Pulses Detected with HETE-2, Publications of the Astronomical Society of Japan 62 (2010) 487.

[10] Ukwatta, T. N. et al., Spectral Lags and the Lag-Luminosity Relation: An Investigation with Swift BAT Gamma-ray Bursts, ApJ 711 (2010) 1073.

[11] Gehrels, N. et al., The Swift Gamma-Ray Burst Mission, ApJ 621 (2005) 558.

[12] Ukwatta, T. N. et al., The lag-luminosity relation in the GRB source frame: an investigation with Swift BAT bursts, MNRAS 419 (2012) 614.

[13] Bernardini, M. G. et al., Comparing the spectral lag of short and long gamma-ray bursts and its relation with the luminosity, MNRAS 446 (2015) 1129.

[14] Barthelmy, S. D. et al., The Burst Alert Telescope (BAT) on the SWIFT Midex Mission, Space Sci. Rev. 120 (2005) 143.

[15] Salvaterra, R. et al., A Complete Sample of Bright Swift Long Gamma-Ray Bursts. I. Sample Presentation, Luminosity Function and Evolution, ApJ 749 (2012) 68.

[16] D’Avanzo, P., et al., A complete sample of bright Swift short Gamma-Ray Bursts

[17] Nava, L. et al., A complete sample of bright Swift long gamma-ray bursts: testing the spectral-energy correlations, MNRAS 421 (2012) 1256. 